\title{
Single-crystal organic field-effect transistors based on dibenzo-tetrathiafulvalene
}

\author{
M. Mas-Torrent ${ }^{\mathrm{a})}$ and P. Hadley \\ Kavli Institute of NanoScience, University of Technology, Lorentzweg 1, 2628 CJ Delft, The Netherlands
}

S. T. Bromley

Departament de Química Física \& Centre Especial de Recerca en Química Teorica, Universitat de Barcelona \& Parc Científic de Barcelona, C/Martí i Franquès 1, 08028 Barcelona, Spain

N. Crivillers, J. Veciana, and C. Rovira ${ }^{\text {b) }}$

Institut de Ciència de Materials de Barcelona(CSIC), Campus UAB, 08193 Cerdanyola, Spain

(Received 23 July 2004; accepted 9 November 2004; published online 28 December 2004)

\begin{abstract}
We report on the fabrication and characterization of field-effect transistors based on single crystals of the organic semiconductor dibenzo-tetrathiafulvalene (DB-TTF). We demonstrate that it is possible to prepare very-good-quality DB-TTF crystals from solution. These devices show high field-effect mobilities typically in the range $0.1-1 \mathrm{~cm}^{2} / \mathrm{V} \mathrm{s}$. The temperature dependence was also studied revealing an initial increase of the mobility when lowering the temperature until it reached a maximum, after which the mobility decreased following a thermally activated behavior with activation energies between 50 and $60 \mathrm{meV}$. Calculations of the molecular reorganization energy and intermolecular transfer integrals for this material were also performed and are in agreement with the high mobility observed in this material. (C) 2005 American Institute of Physics. [DOI: $10.1063 / 1.1848179]$
\end{abstract}

The improved electronic performance of organic fieldeffect transistors (OFETs) over the last few years has shown great potential for a wide range of functional applications where low-cost, light-weight, flexibility, and large-area coverage are required. ${ }^{1}$ Although most previous studies on OFETs have focused on the fabrication and improving the quality of organic thin films, ${ }^{2-5}$ currently, a few groups are devoting their efforts to the preparation of single-crystal OFETs as they typically show higher charge carrier mobilities because of their high molecular order. Crystals of oligoacene $^{6-10}$ and thiophene ${ }^{11}$ derivatives have been studied and OFET mobilities of up to $15 \mathrm{~cm}^{2} / \mathrm{V} \mathrm{s}$ have been reported for rubrene crystals. ${ }^{7}$ In all these cases, the samples were prepared from the vapor phase in order to obtain high purity materials and/or because of the low solubility of these materials in common organic solvents. In this letter, we report on the preparation of single crystal OFETs based on an organic semiconductor, dibenzo-tetrathiafulvalene [DB-TTF, Fig. 1(a)]. Moreover, we show that it is possible to prepare good quality DB-TTF crystals with very high mobilities from solution, which makes this material very interesting for potential applications in low-cost electronics.

Very recently, we studied the correlation between crystal structure and mobility in single-crystal OFETs based on tetrathiafulvalene derivatives. ${ }^{12}$ A correlation between the mobilities and the different investigated crystal structures was observed. This was corroborated by density functional (DF) calculations of the molecular reorganization energies $\left(\lambda_{\text {reorg }}\right)^{13}$ and the maximum intermolecular transfer integrals. ${ }^{14}$ It was observed that the molecules showing the best performance for OFETs crystallize forming uniform stacks of almost planar molecules in a herringbone pattern.

\footnotetext{
${ }^{a)}$ Electronic mail: marta@qt.tn.tudelft.nl

${ }^{b)}$ Electronic mail: cun@icmab.es
}

In addition, these molecules were the ones showing higher intermolecular transfer integrals and lower reorganization energies. In particular, dithiophene-tetrathiafulvalene [DT-TTF, Fig. 1(a)] exhibited a mobility of up to $1.4 \mathrm{~cm}^{2} / \mathrm{V} \mathrm{s}{ }^{15}$

DB-TTF seems, a priori, a very promising candidate molecule to study for the preparation of OFETs as, like DTTTF, it is symmetric and completely conjugated (i.e., alternation of single and double bonds). Also, DB-TTF crystallizes in a similar way to DT-TTF forming stacks of planar molecules with an interplanar distance of $3.948 \AA$ [Fig. 1(b)]. In addition, the calculated highest occupied molecular orbital (HOMO) energy levels (see details to follow) in DB-

(a)

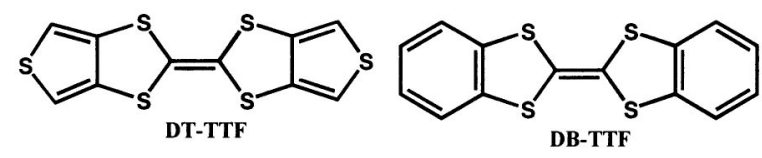

(b)

(c)
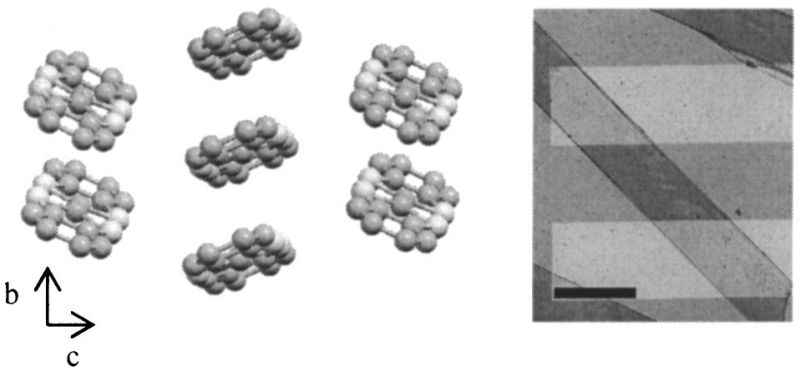

FIG. 1. (a) Molecular structure of dithiophene-tetrathiafulvalene (DT-TTF) and dibenzo-tetrathiafulvalene (DB-TTF). (b) Crystal structure of DB-TTF viewed along the $a$ axis. (c) Single-crystal of DB-TTF on prefabricated gold electrodes. The scale bar corresponds to $100 \mu \mathrm{m}$. 
TTF $(4.88 \mathrm{eV})$ and DT-TTF $(4.94 \mathrm{eV})$ are very similar, which was experimentally confirmed by cyclic voltamperometry measurements of DB-TTF. The experiment showed two separate reversible one-electron oxidations with $E_{1 / 2}^{1}$ and $E_{1 / 2}^{2}$ of 0.62 and $0.97 \mathrm{~V}$, respectively (in $\mathrm{CH}_{3} \mathrm{CN} / 0.1 \mathrm{M} \mathrm{TBAPF}_{6}$ vs SCE) very similar to the values found for DT-TTF in the same conditions $\left(E_{1 / 2}^{1}=0.67 \mathrm{~V}\right.$ and $\left.E_{1 / 2}^{2}=0.97 \mathrm{~V}\right)$.

We performed DF calculations, at a $6-31 \mathrm{G}(d, p)$ / B3LYP ${ }^{16}$ level of theory using GAUSSIAN $98,{ }^{17}$ to estimate the $\lambda_{\text {reorg }}$ of the isolated DB-TTF molecule in the planar conformation found in the crystal. ${ }^{12-18} \mathrm{~A} \lambda_{\text {reorg }}$ value of $0.248 \mathrm{eV}$ was found for this molecule, which is close to the one reported for the best performing molecule in single-crystal OFETs (DT-TTF, $\lambda_{\text {reorg }}=0.238 \mathrm{eV}$ ) in the studied sequence of crystals of molecules of this family. ${ }^{12}$ Previously, we demonstrated that an additional drop in the $\lambda_{\text {reorg }}$ value of DTTTF is observed if one considers the effect of the local crystalline environment, rather than using an isolated molecular representation, helping to explain the observed high mobilities. ${ }^{18}$ This effect is also expected to occur in DB-TTF. The maximum intermolecular electronic coupling, estimated via the calculation of the transfer integrals, ${ }^{19}$ was also calculated for DB-TTF giving a value of $0.037 \mathrm{eV}(0.034 \mathrm{eV}$ for DT-TTF). It therefore could be expected that DB-TTF, due to its similar characteristics to DT-TTF, might be an interesting material to study for the preparation of OFETs.

DB-TTF was synthesized as previously reported ${ }^{20}$ and purified by recrystallization in chlorobenzene. The electrodes ( $4 \mathrm{~nm}$ of Ti, acting as a sticking layer, covered by $26 \mathrm{~nm}$ of $\mathrm{Au})$ were fabricated by e-beam lithography on a silicon wafer with a 200-nm-thick oxide. A warm solution of DB-TTF in chlorobenzene was then poured over the electrodes and the solvent was allowed to evaporate slowly. This resulted in the formation of transparent long regular plate-like crystals, some of which connected two of the microfabricated electrodes [Fig. 1(c)]. In some cases, the formation of long crystalline dendrites was also observed. The long axis of the crystals was determined to be the stacking direction (the crystallographic $b$ axis).

Transport measurements were carried out using a Probe Station Microscope coupled to an ADwin Gold external data acquisition system in air. The electrical characterization of the devices was performed by measuring the source-drain current, $I_{S D}$, while sweeping the applied source-drain voltage, $V_{S D}$, across the two electrodes for different gate voltages, $V_{G}$, applied to the silicon substrate. Figure 2(a) shows the outcome of the measurements performed on the crystal shown in Fig. 1(c). The resulting graph is typical of a $p$-type semiconductor: the current increases with increasing negative gate voltage. The investigated OFETs were stable and all the data could be reproduced after several weeks.

The current-voltage characteristics could be modeled with

$$
I_{S D}=(W C \mu / L)\left(V_{G}-V_{T}-V_{S D} / 2\right) V_{S D},
$$

where $C$ is the capacitance per unit area of the gate, $V_{T}$ the threshold voltage, $W$ and $L$ the width and length of the crystal between the source and drain electrodes, respectively, and $\mu$ the mobility that can be calculated in the linear regime from

Downloaded 13 Aug 2010 to 131.180.130.114. Redistribution subject to AIP license or copyright; see http://apl.aip.org/about/rights_and_permissions

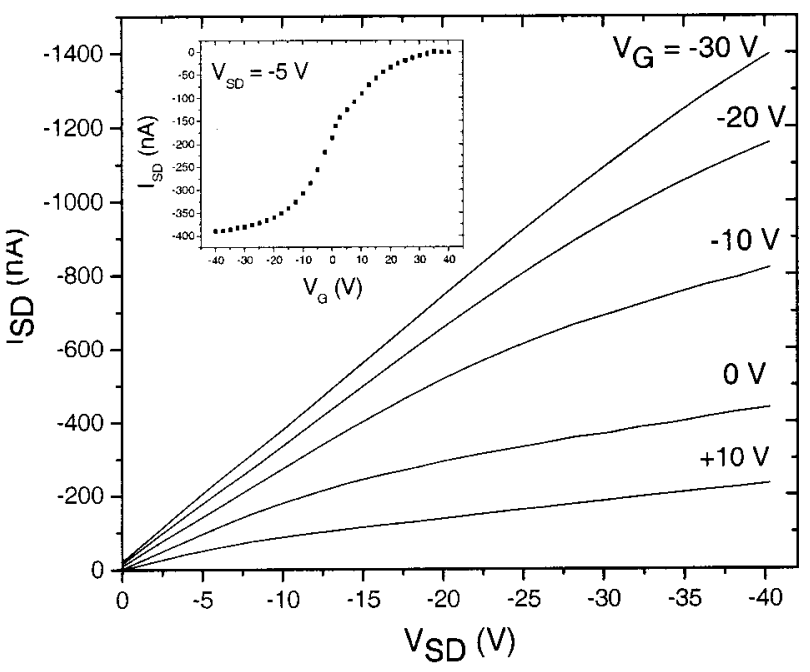

(a)

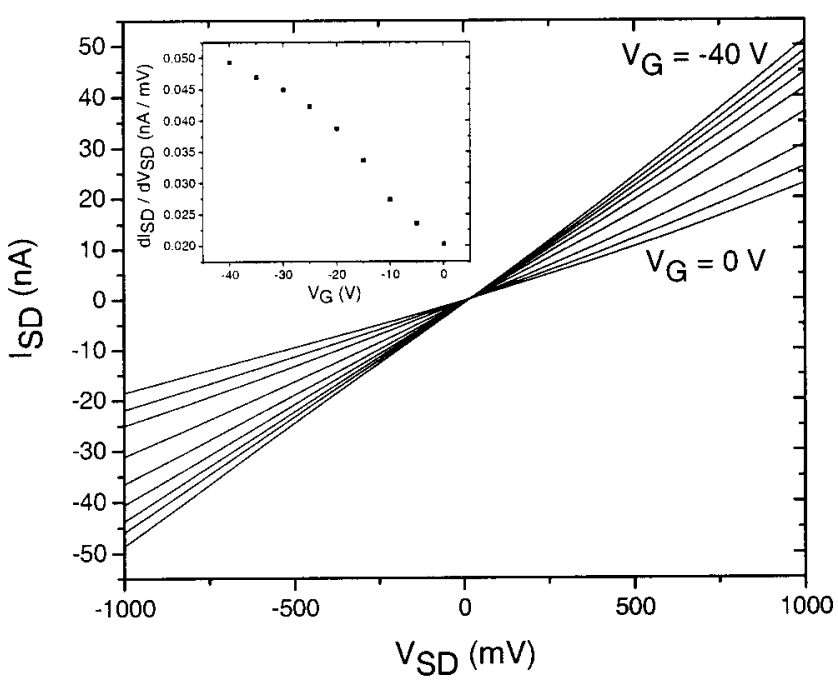

(b)

FIG. 2. (a) $I_{S D}$ vs $V_{S D}$ at different $V_{G}$ measured for the crystal shown in Fig. $1(\mathrm{c})$, which has a length $(L)$ and width $(W)$ of 130 and $70 \mu \mathrm{m}$, respectively. In the inset the transfer characteristics of this device at $V_{S D}=-5 \mathrm{~V}$ are shown. (b) $I_{S D}$ vs $V_{S D}$ at $V_{G}(0,-5,-10,-15,-20,-25,-30,-35$, and $-40 \mathrm{~V}$ ) for a crystal with $L=280 \mu \mathrm{m}$ and $W=15 \mu \mathrm{m}$. In the inset of this graph the conductance $\left(d I_{S D} / d V_{S D}\right)$ vs $V_{G}$ is plotted.

$$
\mu=\frac{\partial^{2} I_{S D}}{\partial V_{S D} \partial V_{G}} \frac{L}{C W} .
$$

This formula neglects the influence of contact resistances and charge traps in the gate oxide and, therefore, provides a lower limit on the device mobility. ${ }^{21}$ The mobility of this device calculated in the range $0 \geqslant V_{G} \geqslant-20 \mathrm{~V}$ was found to be $0.1 \mathrm{~cm}^{2} / \mathrm{V} \mathrm{s}$ and the ON/OFF ratio was of the order of $10^{6}$.

We characterized 18 OFET devices, of which 9 exhibited mobility values larger than $0.01 \mathrm{~cm}^{2} / \mathrm{V} \mathrm{s}$ and 6 higher than $0.1 \mathrm{~cm}^{2} / \mathrm{V} \mathrm{s}$. The highest mobility found was as high as $1.0 \mathrm{~cm}^{2} / \mathrm{V} \mathrm{s}$. The $I_{S D}-V_{S D}$ characteristics of this crystal in the linear regime are shown in Fig. 2(b). From this graph, we can extract the dependency of the conductance $\left(d I_{S D} / d V_{S D}\right)$ with $V_{G}$ [see inset Fig. 2(b)].

The measured threshold voltage $V_{T}$ of these devices is always positive (in the range $10-100 \mathrm{~V}$ ), indicating that there is conduction even without applying a gate voltage. 


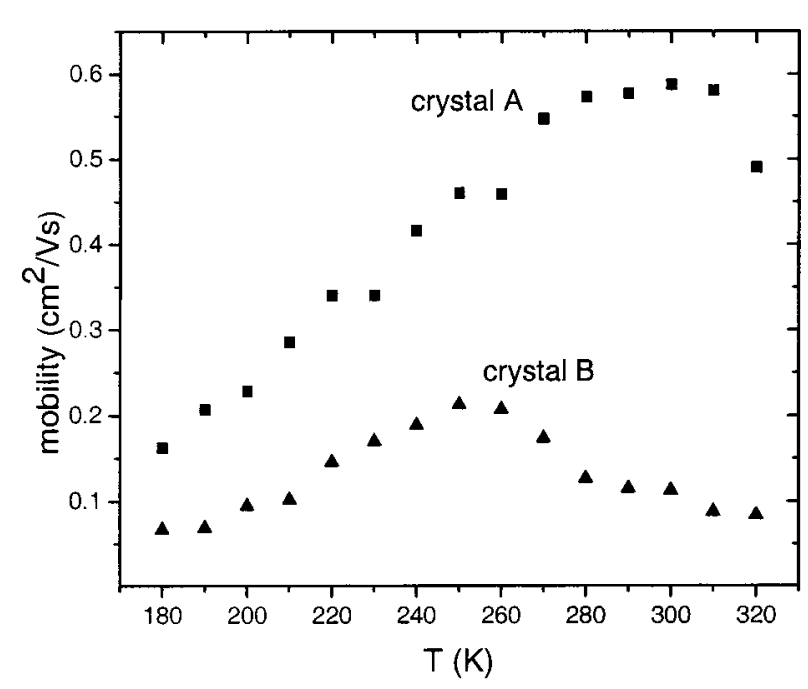

FIG. 3. Field-effect mobility vs temperature for two different single-crystal OFETs of DB-TTF.

This might be due to some unintentional doping and/or to charge accumulated at the interface between the crystal and the substrate. We noted that by annealing one of the samples at about $90{ }^{\circ} \mathrm{C}$ for $20 \mathrm{~min}$, a slightly higher mobility and a $V_{T}$ of only $1 \mathrm{~V}$ was obtained. The annealing step may have driven off solvent initially in the crystal or at the interface between the crystal and the substrate.

We studied the temperature dependence of the mobility in the range 320-180 K for two different OFETs (Fig. 3). In both crystals, the mobility initially increased with decreasing temperature until a maximum was reached, after which the mobility decreased following a thermally activated behavior. Similar trends have been observed for high-mobility OFETs based on pentacene, tetracene, and rubrene. ${ }^{8,22,23}$ Thus, this nonmonotonous temperature dependence is an indication of the good quality of the crystals. The mobility drop at low temperature resembles the prediction of Holstein's model for thermally activated hopping transport. ${ }^{24}$ In this regime, the mobility was fit to the expression $\mu \alpha \exp \left(-E_{a} / K_{B} T\right)$ to determine the activation energies, $E_{a}$, which were found to be 51 and $56 \mathrm{meV}$ for crystals $\mathrm{A}$ and $\mathrm{B}$, respectively. Previously, ${ }^{8}$ this type of temperature dependence was related to a model which assumes the presence of shallow traps and is modeled by $\mu \alpha T^{n} \exp \left(-E_{t} / K_{B} T\right)$, where $E_{t}$ is the trapping depth. ${ }^{25}$ This model predicts that the temperature dependence of the effective mobility should reveal a maximum, $T_{m}$, dependent on the density of traps and the trapping depth. In the high-temperature region for $T>T_{m}$, the microscopic mobility would be dominant and, for $T<T_{m}$, the exponential term of thermally activated mobility would prevail. Thus, the shifting of $T_{m}$ to lower temperatures would indicate a decrease in $E_{t}$ and trap density. Our measurements, therefore, point to lower $E_{t}$ and trap density in crystal B, although crystal A exhibits higher mobility at room temperature (see Fig. 3). However, further systematic temperature measurements are required to fully understand the transport mechanisms in these organic crystals.
In conclusion, we showed here that DB-TTF is a very promising molecule for the preparation of single-crystal OFETs. The devices based on this molecule display very high mobilities of the order of $0.1-1 \mathrm{~cm}^{2} / \mathrm{V} \mathrm{s}$. This material could also be interesting as solution-processable transparent conductor. The high performance of the devices together with the nonmonotonous temperature dependence demonstrates that it is possible to prepare very good-quality crystals of this material from solution, which opens new perspectives for applications in low-cost electronics.

This work was supported by EU by the 6FP NAIMO Integrated Project No NMP4-CT-2004-500355, DGI, Spain (Project No. BQU2003-00760) and DGR; Generalitat de Catalunya (Centre de Referencia CeRMAE and Project 2001SG00362). M.M.-T. also thanks DGR for a nanotechnology fellowship.

${ }^{1}$ S. R. Forrest, Nature (London) 428, 911 (2004).

${ }^{2}$ C. D. Dimitrakopoulos and P. R. L. Malenfant, Adv. Mater. (Weinheim, Ger.) 14, 99 (2002).

${ }^{3}$ Y.-Y. Noh, J.-J. Kim, Y. Yoshida, and K. Yase, Adv. Mater. (Weinheim, Ger.) 15, 699 (2003).

${ }^{4}$ H. Yanagi, Y. Araki, T. Ohara, S. Hotta, M. Ichikawa, and Y. Taniguchi, Adv. Funct. Mater. 13, 767 (2003).

${ }^{5}$ G. H. Gelink, T. C. T. Geuns, and D. M. De Leeuw, Appl. Phys. Lett. 77, 1487 (2000).

${ }^{6}$ V. Podzorov, S. E. Sysoev, E. Loginova, V. M. Pudalov, and M. E. Gershenson, Appl. Phys. Lett. 83, 3504 (2003).

${ }^{7}$ V. C. Sundar, J. Zaumseil, V. Podzorov, E. Menard, R. L. Willett, T. Someya, M. E. Gershenson, and J. A. Rogers, Science 303, 1644 (2004).

${ }^{8}$ R. W. I. De Boer, T. M. Klapwijk, and A. F. Morpurgo, Appl. Phys. Lett. 83, 4345 (2003).

${ }^{9}$ V. Y. Butko, X. Chi, D. V. Lang, and A. P. Ramirez, Appl. Phys. Lett. 83, 4773 (2003).

${ }^{10}$ O. D. Jurchescu, J. Baas, and T. T. M. Palstra, Appl. Phys. Lett. 84, 3061 (2004).

${ }^{11}$ M. Ichikawa, H. Yanagi, Y. Shimizu, S. Hotta, N. Suganuma, T. Koyama, and Y. Taniguchi, Adv. Mater. (Weinheim, Ger.) 14, 1272 (2002).

${ }^{12}$ M. Mas-Torrent, P. Hadley, S. T. Bromley, X. Ribas, J. Tarrés, M. Mas, E. Molins, J. Veciana, and C. Rovira, J. Am. Chem. Soc. 126, 8546 (2004).

${ }^{13}$ The reorganization energy in a hole-hopping material is defined as the sum of the geometrical relaxation energies of one molecule upon going from the neutral-state geometry to the charged-state geometry and the neighboring molecule upon going the inverse process $\left[A(0)+A^{\prime}(+) \rightarrow A(+)\right.$ $\left.+A^{\prime}(0)\right]$. See for example: M. Malagoli and J. L. Brédas, Chem. Phys. Lett. 327, 13 (2000).

${ }^{14}$ J. L. Brédas, J. P. Calbert, D. A. da Silva Filho, and J. Cornil, Proc. Natl. Acad. Sci. U.S.A. 99, 5804 (2002).

${ }^{15}$ M. Mas-Torrent, M. Durkut, P. Hadley, X. Ribas, and C. Rovira, J. Am. Chem. Soc. 126, 984 (2004).

${ }^{16}$ A. D. Becke, J. Phys. Chem. 98, 5648 (1993).

${ }^{17}$ M. J. Frisch et al., GAUSSIAN 98, Revision A.9, Gaussian, Inc. (Pittsburgh, PA, 1998).

${ }^{18}$ S. T. Bromley, M. Mas-Torrent, P. Hadley, and C. Rovira, J. Am. Chem. Soc. 126, 6544 (2004).

${ }^{19}$ The electronic coupling between adjacent DB-TTF molecules in the crystal was estimated by the method described in Ref. 14.

${ }^{20}$ M. Mizumo and M. P. Cava, J. Org. Chem. 43, 416 (1978).

${ }^{21}$ L. Bürgi, T. J. Richards, R. H. Friend, and H. Sirringhaus, J. Appl. Phys. 94, 6129 (2003)

${ }^{22}$ S. F. Nelson, Y.-Y. Lin, D. J. Gundlach, and T. N. Jackson, Appl. Phys. Lett. 72, 1854 (1998).

${ }^{23}$ R. W. I. De Boer, M. E. Gershenson, A. F. Morpurgo, and V. Podzorov, Phys. Status Solidi A 201, 1302 (2004).

${ }^{24}$ T. Holstein, Ann. Phys. (N.Y.) 8, 325 (1959).

${ }^{25}$ E. A. Silinsh and V. Čápek, Organic Molecular Crystals (AIP, Woodbury, 1994). 